REVESCO. Revista de Estudios Cooperativos

ISSN: $1885-8031$

\title{
El Emprendimiento Social en el marco de la Alianza del Pacífico
}

\author{
Roberto García Alonso ${ }^{1}$, Ulf Thoene ${ }^{2}$, Ana María Figueroa ${ }^{3}$ y Edwin Murillo Amaris ${ }^{4}$
}

Recibido: 3 de abril de 2019 / Aceptado: 1 de octubre de 2019 / Publicado: 23 de enero de 2020

Resumen. En los últimos años ha habido un creciente interés por el emprendimiento social aun cuando existe cierta confusión o controversia sobre su significado. La mayoría de la literatura reconoce la importancia de la articulación de diversos factores dentro de un ecosistema favorable. Pese a esto, aún son muy escasos los estudios relativos a esta materia en el ámbito latinoamericano. Es por ello por lo que este artículo vincula la importancia de la Alianza del Pacifico, como espacio de integración regional para la promoción de este tipo de iniciativas. A partir del análisis de sus documentos oficiales y de entrevistas a responsables de toma de decisiones se identificaron factores que dentro del marco de la Alianza del Pacífico facilitan la consolidación de procesos de emprendimiento social.

Palabras clave: Chile; Colombia; Integración regional; México; Perú; Regionalismo.

Claves Econlit: F02; F63; L31; O54.

\section{[en] Social Entrepreneurship in the Pacific Alliance}

\begin{abstract}
In recent years there has been a growing interest in social entrepreneurship even though there is some confusion or controversy about its meaning. Most of the literature recognizes the importance of the articulation of various factors within a favorable ecosystem. In spite of this, there are still very few studies related to this subject in Latin America. That is why this article establishes links to the importance of the Pacific Alliance as a regional integration space for the promotion of such initiatives. Based on the analysis of official documents and interviews with decision makers, factors were identified that, within the framework of the Pacific Alliance, facilitate the consolidation of social entrepreneurship processes.
\end{abstract}

Keywords: Chile; Colombia; Mexico; Peru; Regionalism; Regional integration.

Sumario. 1. Introducción. 2. Marco teórico-conceptual. 3. Metodología. 4. La importancia del Emprendimiento Social para la Alianza del Pacifico. 5. Ecosistema de Emprendimiento Social desde la Alianza del Pacifico. 6. Explorando más allá del discurso oficial de la Alianza del Pacifico. 7. Conclusiones. 8. Referencias bibliográficas.

Cómo citar: García Alonso, R.; Thoene, U.; Figueroa, A.M.; Murillo Amaris, E. (2020) El Emprendimiento Social en el marco de la Alianza del Pacífico. REVESCO. Revista de Estudios Cooperativos, vol. 133, e67341. https://dx.doi.org/10.5209/REVE.67341.

\section{Introducción}

Teniendo en cuenta que el trabajo conjunto entre actores permite el fortalecimiento de capacidades para la reducción de brechas socio-económicas e institucionales entre diferentes países, la integración regional se ha consolidado como estrategia de desarrollo para los distintos Estados (AECID, 2015).

En las últimas décadas del siglo XX, simultáneo a un fenómeno extendido de transiciones democráticas, se configura un ambiente de altos costos humanos y ambientales, debido a los rigurosos programas de ajuste estructural promovidos por el Fondo Monetario Internacional y el Banco Mundial (ul Haq, 1995, 2018a, 2018b). Como consecuencia, se incrementa el interés por buscar alternativas que tengan en cuenta los sectores más vulnerables de la sociedad. Así pues, el desarrollo humano adquiere importante relevancia desde la década de los años ochenta.

1 Universidad de La Sabana, Bogotá, Colombia

Dirección de correo electrónico: roberto.garcia@unisabana.edu.co

2 Universidad de La Sabana, Bogotá, Colombia

Dirección de correo electrónico: ulf.thoene@unisabana.edu.co

3 Socialab, Bogotá, Colombia

Dirección de correo electrónico: anamariafigueroagarcia@gmail.com

4 Universidad Santiago de Cali, Cali, Colombia

Dirección de correo electrónico: edwin.murillo00@usc.edu.co 
En concordancia con este nuevo paradigma, "durante las últimas dos décadas ha crecido exponencialmente el emprendimiento social, con altos índices de eficacia en la solución de problemas sociales alrededor del mundo" (Guzmán y Trujillo, 2008:119). Sus encargados se reconocen como agentes de cambio, pues utilizan la innovación como instrumento para la creación de valor social sostenible (Chen et al., 2016). Su objetivo, desde una estrategia bottom-up, es empoderar a las personas para que puedan ser responsables de su desarrollo.

Por otro lado, en 2011 surge la Alianza del Pacífico (AP, 2012a) como mecanismo de integración económico-comercial -el más reciente en Latinoamérica- entre Chile, Colombia, México y Perú (Echebarría y Estevadeordal, 2014; Thoene et al., 2017). Entre otras cosas, parte de su espacio y esfuerzos los dedica al emprendimiento y la innovación, reconociendo que la región se encuentra rezagada en estos ámbitos y, en esta medida, siendo un obstáculo permanente para alcanzar transformaciones productivas más equitativas (Rosales, 2012).

Teniendo en cuenta lo anterior, surge la duda sobre cómo la Alianza del Pacífico (AP) puede funcionar como plataforma para el impulso y la implementación del Emprendimiento Social (ES) dentro de sus países miembros. Así pues, el objetivo principal de este artículo es identificar los factores que pueden facilitar la consolidación de procesos de ES en la AP, con el fin de determinar si ésta constituye una plataforma de promoción e implementación de este tipo de actividades dentro de sus países miembros, a pesar de que funcionan bajo lógicas de desarrollo disímiles.

En este sentido, el presente documento desarrolla sus bases teóricas y conceptuales a partir de una discusión sobre el regionalismo y la integración regional latinoamericana, y alrededor del ES. Posteriormente, describe las herramientas metodológicas utilizadas en esta investigación, presenta los resultados de dichas estrategias, y complementa con la exploración del panorama de ES de la región y los instrumentos que la misma tiene para impulsar este tipo de procesos.

\section{Marco teórico-conceptual}

\subsection{Integración regional y regionalismo latinoamericano}

En un contexto de post Guerra Fría de orden multilateral, gobernanza multipolar y sistema de regiones (Vivares y Calderón, 2014:6), donde a pesar de las limitaciones por las dinámicas de cada contexto el Estado sigue siendo espacio de concurrencia de donde concurren tendencias de desarrollo (Farrell et al., 2005:321), entendiendo que este concepto se concibe como un producto del orden del poder mundial, regional y nacional, relacionado con condiciones heredadas o recientemente creadas que determinan la calidad de vida de la sociedad (Payne, 2004), pero con una fuerte limitación por las dinámicas de cada contexto. En este caso, se entiende el regionalismo como un "cuerpo de ideas, valores y proyectos políticos que contribuyen a la creación, mantenimiento o transformación de un tipo de [...] orden mundial" (Vivares y Calderón, 2014:4), socialmente construidas bajo lineamientos de desarrollo y cuyos actores interactúan con un alto grado de interdependencia (Nye, 1968:448), y, además, se asocia usualmente a los programas de política pública y las estrategias de construcción de estructuras institucionales regionales, sin que esto implique siempre la cesión de autoridad estatal.

De la mano con el regionalismo aparece la integración regional como un proceso de naturaleza dinámica que aborda sus quehaceres como medio para el progreso de las sociedades bajo un enfoque consensuado y voluntario de transformación social consensuado y voluntario, en el que un grupo de unidades sociales tienden a asociarse de manera parcial o absoluta total en una unidad mayor (Gamboa y Fernández, 2013:731), bajo intereses comunes. El caso latinoamericano fue impulsado por la dependencia económica de la región, direccionado hacia el desarrollo nacional y la consolidación de la soberanía interna (Betancourt y Prieto, 2014:85).

Aquí se identifican tres etapas: 1) el regionalismo cerrado, enmarcado en el modelo de Industrialización por Sustitución de Importaciones; 2) las reformas de ajuste estructural del Consenso de Washington, que representaron un giro hacia un regionalismo abierto (Betancourt y Prieto, 2014). Esta última involucraba la estabilización macroeconómica, la facilitación del comercio, la armonización de normas comerciales, así como las regulaciones internas y estándares (Forero, 2010), generó una mayor liberalización de sus mercados, una desregulación económica y la atracción del capital extranjero (Cancino y Albornoz, 2007). Es decir, una integración más abierta, competitiva, donde el Estado tendía a ceder su primacía a las leyes del mercado (Vieira, 2007). 3) En respuesta a sus consecuencias se gestaron posturas integracionistas alternativas - regionalización poshegemónica - a partir del cambio de orientación política en algunos países de América Latina asumiendo un carácter crítico con el discurso hegemónico del libre comercio (BriceñoRuiz, 2014; Briceño-Ruiz y Morales, 2017).

Como resultado, se instaura una estructura multinivel regionalista e intergubernamental, que además permite la inclusión de actores no estatales en la toma de decisiones y funciona a partir de consensos 
políticos, en vez de cesión de soberanía a instancias supranacionales (contrario al modelo europeo) (Vivares y Calderón, 2014:11). Además de la diversificación de la agenda de desarrollo. La AP es un ejemplo claro de lo mencionado. Se estructura de esta forma, en la interrelación de dos regiones concretas como el AsiaPacífico y América Latina, un caso de gobernanza multinivel que "permite el avance hacia la configuración de espacios de gestión multilateral en los que tienen cabida diversos actores, sin que esto signifique la pérdida de protagonismo del Estado, lo que aumenta la capacidad de respuesta de los andamiajes de gobernanza a las demandas de una sociedad que ha trascendido los límites del Estado-nación y que articula, cada vez más, en redes regionales y globales" (Pastrana et al., 2014).

\subsection{La Alianza del Pacifico}

La AP surgió el 28 de abril de 2011 -Constituida formal y jurídicamente con la suscripción del Acuerdo Marco el 6 de junio de 2012 (AP, 2012a)- como mecanismo de integración regional, a través de una alianza económico-comercial y estratégica (Fernández de Soto, 2015). Ésta pretende recurrir a herramientas de participación y consenso para la consolidación de un área de integración profunda, a través de la liberalización del comercio de bienes, servicios, movimiento de capitales y personas. Además, tiene la motivación de promover mayor crecimiento, desarrollo y competitividad de las economías de sus países miembros, como medio para superar las desigualdades socioeconómicas (Foxley y Meller, 2014; Thoene y Gaitán, 2019).

Cada uno de ellos tiene un fuerte compromiso con la economía de libre mercado y el fortalecimiento de la inversión privada y extranjera, como motores de crecimiento económico (Betancourt y Prieto, 2014). Adicionalmente, pretende fortalecer la acción conjunta entre las agencias de promoción de exportaciones de Chile, Colombia, México, y Perú -ProChile, ProColombia, ProMéxico, y PromPerú- y la cooperación para el mejoramiento de la competitividad e innovación (AP, 2012b). Todo esto constituye una matriz operativa pragmática, incrementando la eficacia de los procesos de discusión, toma de decisiones, evaluación y aprobación.

Aunque la AP surge en paralelo con la tercera ola del regionalismo latinoamericano, tiene mucha más cercanía con el regionalismo abierto de los años noventa (Pastrana, 2015). No obstante, Fernández de Soto (2015) considera que este proyecto debería ser ubicado dentro de un "regionalismo interdependiente" fundamentado en una agenda multidimensional. Ésta concibe la integración como espacio natural para la consolidación de la región como actor internacional con identidad propia, pero con estructuras no excluyentes de inserción internacional. En este orden de ideas, el regionalismo interdependiente es más flexible en la medida en que acepta velocidades distintas para las prácticas de difícil consenso y compromisos vinculantes para los elementos de coincidencia, contando con mayor legitimidad dada la inclusión de empresarios y de la sociedad civil (Fernández de Soto, 2015).

Este "regionalismo interdependiente" fundado en una agenda multidimensional, tal como ha sido citado desde Fernández de Soto (2015), posibilita la interacción entre dos regiones claramente definidas (AsiaPacífico y América Latina: Chile, Perú, Colombia y México) que disponen potencialidades para un diseño estratégico de decisiones públicas en lo referente al ES, como se probará a lo largo de este texto. Para la región de América Latina, las temáticas sociales son un eje transversal que acompaña la vida socio-política, económica y cultural en sus decisiones públicas. En este sentido, "esta nueva manera de gobernarnos internacionalmente, fundada en el establecimiento de redes de cooperación y apoyo, abriendo el marco de lo regional y lo subregional como espacios de referencia, tiene en lo social un instrumento clave de oportunidades de desarrollo en materia de garantizar los principios de coordinación, gerencia y reglamentación de lo colectivo", como lo expresan Flemes y Radseck (2012). Es ahí donde el ES se define como eje articulador en una apuesta como entre la AP y el Asia-Pacífico.

\subsection{Emprendimiento Social}

El paradigma de desarrollo humano aboga por la creación de "un ambiente que permita a las personas disfrutar de una vida larga, saludable y llena de creatividad" (ul Haq, 2018a). Esto a partir de la ampliación del horizonte de oportunidades y posibilidades de todas las personas, con base en el incremento de la riqueza humana, y teniendo como pilares fundamentales a la igualdad, la sustentabilidad, la productividad y el empoderamiento (ul Haq, 2018b). Aquí el crecimiento económico se encuentra subordinado a "la gestación de voluntades sociales que aspiran a la participación, a la autonomía y a una utilización más equitativa de los recursos disponibles" (Max-Neef, 1986:62).

De manera más específica, Max-Neef (1986) propone estrategias de desarrollo autodependiente, donde las personas tienen protagonismo real y cuyos resultados para la satisfacción de las necesidades fundamentales son sinérgicos. La autodependencia es eje vertebral, al ser interdependencia horizontal o "un proceso capaz de fomentar la participación en las decisiones, la creatividad social, la autonomía política, la justa distribución de la riqueza y la tolerancia frente a la diversidad de identidades" (Max-Neef, 1986:57). 
Por su naturaleza, el ES se considera como una estrategia de desarrollo bottom-up, que pretende alcanzar la generación de recursos destinados para el desarrollo desde las organizaciones locales (Max-Neef, 1986). Usualmente, se define como una actividad emprendedora, cuya generación de ingresos es destinada a la búsqueda y consecución simultánea de metas económicas, sociales y ambientales, por medio de mecanismos de disciplina empresarial e innovación (Santos, 2009). Presenta características del sector privado -utilizando el mercado para generar innovación y productividad-, el sector público o gubernamental -respondiendo a las fallas del mercado proveyendo bienes y servicios públicos-, y el sector sin ánimo de lucro -comprometiendo con los individuos en la redistribución de los recursos de los elementos más ricos de la sociedad a los menos pudientes-, con la intención de crear efectos de impacto múltiple, demostrando ser un agente único (Santos, 2009).

En concordancia con lo anterior, el ES está regido por el beneficio de grupos sociales y la inclusión de sistemas de cooperación (Santos, 2009). Su misión es crear valor social sin limitarse a la disponibilidad de recursos e incluye un profundo, focalizado y vanguardista conocimiento del área y situación social a intervenir (Dees, 2011). En este proceso, se persiguen persistentemente nuevas oportunidades de creación, adaptación y aprendizaje, donde la innovación social resulta elemental, pues permite alteraciones permanentes en el sistema, mediante iniciativas creativas basadas en el mercado que generan crecimiento y oportunidades, en la medida en que van solucionando un problema social o ambiental determinado. Este mecanismo no es exclusivo de un actor específico, sino que surge de puntos en común entre las diferentes partes (Buckland y Murillo, 2014). Por tales razones, los emprendedores sociales también son agentes de cambio en el sector social.

Ellos se enfocan en las causas subyacentes de los problemas, generando ideas que rompen los parámetros para la generación de soluciones sostenibles y cambios sistémicos que reduzcan o eliminen de manera permanente las necesidades de la población afectada (Reza Noruzi, 2010; Dees, 2011). Aunque su labor está enfocada principalmente en el ámbito local, su impacto puede alcanzar repercusiones a nivel global. En consecuencia, se aborda el ES como proceso o estructura de permanente evolución, en la que se interrelacionan las personas, el contexto, la transacción y la oportunidad, a partir de un eje trasversal: el objetivo social (Chen et al., 2016).

Dada su naturaleza de buscar oportunidades el ES reconoce a la mayoría de externalidades del mercado como oportunidades para crear valor, sin estar determinados por el potencial de apropiación, por la forma jurídica del mismo o por el tipo de objetivo social que se persiga (Lejarriaga y Bel, 2018). En este orden de cosas, el valor social puede manifestarse de muy diferentes formas, con actuaciones respetuosas con el medio ambiente, favoreciendo la inclusión social u otras formas socialmente responsables. Si bien existen fórmulas jurídicas más apropiadas que otras para lograr este tipo de fines, pero estos proyectos e iniciativas pueden acogerse a cualquier modalidad legal posible (Martín, 2018).

Como resultado, el ES adopta lógicas de empoderamiento de los beneficiarios y las partes interesadas, creando, de esta manera, opciones deliberadas y transformándolas en acciones y resultados deseados, en vez de partir del razonamiento tradicional de control (Santos, 2009). De esta manera, se incluyen como parte integral, reduciendo su dependencia hacia la organización y aumentando sus habilidades. Todo esto requiere de un ecosistema favorable que permita -en el corto, mediano y largo plazo- complementariedad e interdependencia entre la organización y su contexto (Vernis y Navarro, 2011). Aquí, el capital social es un factor importante dado que hace referencia a "las relaciones y los flujos de información, así como los recursos necesarios para que este ecosistema esté vivo y operante" (Buckland y Murillo, 2014:38). De esta manera, los emprendimientos sociales pueden interactuar en el mercado en igualdad de condiciones con otros actores.

En contextos sociales particulares que se pueden hallar en las subregiones que configuran la AP y el AsiaPacífico, este elemento del capital social en perspectiva de refuerzo a la estrategia del ES es de vital importancia para que surtan los eficaces y eficientes efectos. Como consecuencia, "el conglomerado de redes sociales, normas y vida asociativa, debe evidenciarse la confianza interpersonal para facilitar la cooperación" (Jordana, 2000; Putnam, 1993) que, desde la perspectiva de esta investigación, acelera los insumos fundantes del ES.

Otro insumo de análisis que no se puede desconocer es el de cohesión social. Se puede configurar una interrelación dialógica entre "desarrollo/sostenibilidad - capital social y cohesión social" como componentes estratégicos del ES. Desde el año 2007, América Latina viene enfatizando desde las reflexiones de la Comisión Económica para América Latina y el Caribe (CEPAL) en esta urgencia, aunque las necesidades sociales son estructurales y datan de siglos de historia. "El desarrollo de las economías [de la región] requiere de una transformación de las estructuras productivas, acompañadas de un proceso intensivo de formación de capital humano" (Machinea, 2006). De ahí la importancia de la existencia de mecanismos de apoyo a los emprendedores, en términos de que los jóvenes adquieran a través del sistema educativo las competencias y habilidades requeridas para aprender (Espada et al., 2018).

"La cohesión social reside en la dialéctica entre integración e inclusión, por un lado, y capital social y ética social, por el otro" (Hopenhayn, 2007). Es un constructo que se gesta entre elementos de estructuras 
sociales e institucionales y de sujetos (individuales y sociales), y las respuestas que esos mismos componentes ofrecen a las realidades en las que las demandas y necesidades se visibilizan e impactan. Entonces, el ES puede ser efecto de toda la interrelación a través de decisiones en un marco de gobernanza multinivel al estilo de la AP. Sin embargo, en el cómo está el quid.

Las variables de este ecosistema funcionan en red para asegurar su sostenimiento (Vernis y Navarro, 2011): 1) formación, investigación y asesoramiento; 2) recursos financieros y humanos; 3) innovación y creatividad; 4) difusión y visibilidad; y, finalmente, 5) apoyo del Estado. Si bien, todas las variables son importantes, el apoyo del Estado tiene un gran valor para la presente investigación en razón de su vinculación con el fenómeno de integración regional de la AP, la provisión de infraestructura, educación y salud pública, y rule of law.

Una vez aceptado dentro del discurso público, el ES adquiere mayor solvencia dentro del marco de las normas sociales y culturales. Ambos actores cumplen con roles que pueden considerarse complementarios, si se tiene en cuenta que uno -con su dinámica bottom-up - puede suplir algunas labores que el otro -desde su dinámica top-down- no puede realizar y viceversa. Sin embargo, estos no son necesariamente dependientes uno del otro.

En otras palabras, el ES encuentra mayor impulso cuando ambos actores actúan como socios en la consecución de un mismo propósito (Stephan et al., 2015). Es evidente entonces la importancia que tiene la inversión pública en este tipo de alternativas. No obstante, las administraciones públicas fallan en esta tarea cuando su apoyo no está acorde con el funcionamiento en red del ecosistema (Santos, 2009).

\section{Metodología}

Para este análisis se utilizó una metodología cualitativa, que incluye herramientas tanto de análisis del discurso, como de análisis de contenido. Complementariamente, se elaboraron entrevistas y se recurrió a la revisión de fuentes primarias y secundarias.

\subsection{Análisis de documentos oficiales}

En un primer momento, la revisión de documentos oficiales de la AP precisó la importancia del ES dentro de este proceso de integración. Además, permitió identificar mecanismos facilitadores de la configuración de un panorama del ES dentro de los cuatro Estados involucrados. Se escogieron catorce documentos que consignan la evidencia de la evolución de la AP y la información acerca de las reglas de juego, los compromisos establecidos y las necesidades o acciones pendientes en función de avanzar en el proceso de integración regional -entre los que se encuentra el Acuerdo Marco, el Protocolo Adicional al Acuerdo Marco, las Declaraciones de todas las Cumbres Presidenciales de la AP y dos declaraciones sobre temas relacionados con Cambio Climático y PYMES-. En este punto, el análisis de contenido se efectuó segmentando datos en unidades de significado, haciendo codificaciones, conteos y análisis, mediante el programa Atlas Ti (Hernández et al., 2014).

Primero, se recurrió a marcas de nube, para encontrar el tema más mencionado, identificando si las palabras 'emprendimiento', 'social' e 'innovación' estaban incluidas. Posteriormente, se hizo una codificación de cada texto, cuyas subcategorías fueron las mismas áreas de trabajo acordadas por los Estados miembros de la AP (AP, 2012c), que, a su vez, fueron clasificadas en tres grandes categorías.

A partir de esto, se desarrollaron redes semánticas, que permitieron ver la relación entre ellas y la naturaleza de dichos vínculos. El uso de este método también fue adecuado para entender los ecosistemas del ES por la interconexión de sus variables. Así, en la red semántica correspondiente, éstas están asociadas entre sí, en constante relación y a manera de ciclo. Todo esto se complementó con matrices de 'co-ocurrencia de códigos', que mostraron las categorías y subcategorías vinculadas entre sí, después de la codificación. De esta forma, fue posible encontrar de manera más explícita las relaciones entre temáticas.

\subsection{Exploración de experiencias que respaldan el discurso oficial}

En esta sección se intentó encontrar vínculos empíricos entre el ES y la AP, por medio de experiencias y personas involucradas en estas dinámicas. Bajo dicha lógica, se revisó el panorama de ES en América Latina, con especial énfasis en los cuatro Estados involucrados. Adicionalmente se realizaron entrevistas semiestructuradas, lo que proporcionó un mayor margen de maniobra para la inclusión y exploración de nuevas cuestiones, significados y áreas de interés; en la medida en que es una herramienta que abre espacios propicios para que los principales interrogantes puedan ser replanteados según las necesidades y rumbo de la conversación (Arksey y Knight, 1999). Finalmente, la revisión de prensa y fuentes primarias y secundarias permitió comprobar el estado actual de los mecanismos identificados en secciones anteriores. 


\section{La importancia del Emprendimiento Social para la Alianza del Pacifico}

Inicialmente, las marcas de nube mostraron el enfoque hacia el libre comercio en los documentos oficiales de la AP, en sintonía con sus principios neoliberales. Se encontró una relevancia baja para las palabras 'social', 'emprendimiento' e 'innovación' -aunque eventualmente se mencionan-.

Respecto a la codificación, la categoría que registra más frecuencias es la económica, seguida por los asuntos sociopolíticos y medioambientales y, por último, los asuntos institucionales y de infraestructura. Sin embargo, la brecha entre los tres tipos de asuntos no es muy amplia. Esto puede ser evidencia de que el proceso de integración de la AP intenta incluir varias dimensiones desde el principio neoliberal.

Por otro lado, lo más destacable del análisis de redes semánticas es la asociación entre 'Innovación' y 'PYMEs' -entendiendo que la AP considera a éstas como motor de creación de empleo y crecimiento económico-. En ese sentido, son fundamentales para el desarrollo de los países miembros, por lo que constituyen un sector significativo en la región, aunque no se encuentran plenamente integradas a las cadenas de valor. El emprendimiento como pieza vital, permite mayor adaptabilidad a las dinámicas del mercado y a las iniciativas innovadoras (Luna, 2012).

Ahora bien, al unir las tres redes semánticas, es notable el papel vertebrador de la cooperación. Gracias a ella es que se logran los acuerdos entre los países miembros y entre la AP y otros actores en todo tipo de temáticas. También se destacan las entidades de promoción, pues prácticamente son la marca de cada país y de la alianza misma.

Todo esto se complementa con la co-ocurrencia de códigos, mostrando que las tres grandes categorías están relacionadas entre sí. Al contrastar la parte económica con la socio-política y medioambiental, sale a la luz el enlace entre 'Innovación' y 'Cultura', el cual se da por la intención de apoyar iniciativas de emprendimiento cultural. También, surge uno entre 'Innovación' y 'Educación' y otro entre 'PYMES' 'Educación', relacionados con el fortalecimiento del capital humano.

\section{Ecosistema de Emprendimiento Social desde la Alianza del Pacífico}

La codificación reveló que existen compromisos, o se han realizado acciones, en todas y cada una de las variables de estudio desde el proceso de integración regional de la AP. Dichos mecanismos identificados pueden encontrarse en casi todas las variables establecidas, demostrando la multidimensionalidad de la agenda de la AP.

Se puede observar que 'Formación, Investigación, Asesoramiento' es la dimensión que más se respalda la organización, mientras que 'Apoyo Gubernamental' es la que menos apoyo recibe. También se destaca que la mayoría de estos mecanismos recibe poca o nula atención en los primeros años de la AP, pues es a partir del 2014 que este tipo de elementos empiezan a adquirir mayor importancia.

Debido a que la complementariedad e interdependencia entre esas variables resulta ser vital para el adecuado funcionamiento del ecosistema, lo presentado hasta ahora no es evidencia suficiente para asegurar que la AP se pueda considerar como plataforma para el emprendimiento social. En primer lugar, porque puede que algunos planes solo estén consignados como compromisos ambiguos, lo cual no garantiza su cumplimiento. Segundo, porque es posible que los mecanismos estén planteados en función de objetivos distintos al del emprendimiento social. Y, finalmente, porque la codificación por sí sola no permite ver si existe relación entre dichas estrategias, y si funcionan de manera independiente o en red.

Ahora bien, para la red semántica de la investigación, se ubicó la innovación y creatividad en su centro, ya que de allí parte cualquier emprendimiento social. El rol de la difusión y visibilidad está asociado con la etapa de expansión, y es relevante para una retroalimentación constante. Por su parte, el apoyo gubernamental ayuda a que el proceso sea mucho más fructífero y eficaz. Lo más importante es que, desde cualquier punto, se regresa siempre a la innovación, al ser la herramienta que garantiza el pragmatismo de sus operaciones por su estructura flexible y de fácil adaptabilidad.

Como se evidenció en la sección anterior, y de acuerdo con lo expuesto en el marco teórico-conceptual, las temáticas de innovación, PYMES, educación y cooperación están fuertemente relacionadas con los procesos de emprendimiento social. Por sus naturalezas e implicaciones, los vínculos más importantes son con las áreas de innovación, PYMES y educación.

La co-ocurrencia en este caso, también mostró un fuerte vínculo con las entidades de promoción, en razón su importancia para el diseño e implementación de eventos como el 'Foro de Emprendimiento e Innovación LAB4+'. Este mecanismo, junto con el Premio Innovación, cuenta con un agregado fundamental para esta investigación: incluye explícitamente al ES o la innovación social dentro de sus discursos, incluso dedica uno de sus espacios a este tipo de iniciativas. 


\section{Explorando más allá del discurso oficial de la Alianza del Pacífico}

Actualmente, América Latina requiere mejorar su sector industrial, así como encontrar alternativas incluyentes de desarrollo que no dependan solo de la actividad económica (Cassar, 2015). Por esto, se destaca la necesidad de estimular la innovación y el ES de la mano con la investigación, las tecnologías emergentes y nuevos modelos empresariales que contemplen la inclusión social a la par con la inclusión financiera (Thoene y Turriago, 2017).

El ES y la innovación son claves en ese proceso. Sin embargo, administraciones públicas atrasadas en términos de innovación, altas tasas de desigualdad y pobreza, sistemas educativos débiles y baja competitividad de las pequeñas y medianas empresas (PYMES), constituyen grandes obstáculos para que la región pueda reconfigurar su visión de desarrollo (Avogadro, 2016). Los presidentes de los países miembros de la AP reconocen dicha situación, lo que ha facilitado la creación e implementación de estrategias conjuntas para que desde los gobiernos se fomenten ecosistemas favorables a tales prácticas (Tolama, 2016).

Latinoamérica hoy pasa por un 'boom' de ES debido a los recurrentes obstáculos sistémicos o coyunturales (López, 2016). Esto, ha generado infinidad de oportunidades que propician las condiciones para que emprendedores sociales latinoamericanos desarrollen gran capacidad de adaptación (López, 2016). No obstante, aún carecen de mecanismos legales que protejan y faciliten su labor. En concordancia, y partiendo del deseo de generar un mayor impacto social y económico, se requiere de una sinergia entre la visión de las administraciones públicas y el ES (Zuñiga, 2016).

Para tener mucho más claro el panorama de Chile, Colombia, y Perú, el reporte mundial sobre emprendimiento social del Global Entrepreneurship Monitor (2015), revela que la prevalencia de la actividad de ES en su etapa start-up y la operativa, entre la población adulta de los primeros tres países, es mucho mayor a la mexicana, e incluso ocupan los niveles más altos entre los países estudiados (Bosma et al., 2015). Esto se ve reflejado, en niveles relativamente saludables.

\subsection{La posición de algunas entidades involucradas}

En primer lugar, se recurrió a Innpulsa Colombia, por ser la unidad por medio de la cual el Gobierno Nacional canaliza sus esfuerzos para el impulso del emprendimiento y la innovación dentro de sus fronteras. Claudio Alberto Moreno, de la Oficina de Innovación y Emprendimiento, comenta que ellos apoyan emprendimientos sociales con potencial de alto impacto (Moreno, 2016). No obstante, no cuentan con programas o directrices enfocadas en este tipo en concreto a la AP. Innpulsa, ha participado en eventos de la $\mathrm{AP}$, como el LAB4+, donde ha expuesto casos de emprendimientos sociales -sin que estos tengan nexos con algún mecanismo de la AP (Moreno, 2016).

Por su parte, Carlos Enrique Olaya, director comercial de la Cámara de la Alianza del Pacífico, reconoce la importancia del emprendimiento social para el desarrollo de los países implicados, asegurando que es un área que debe ser trabajada desde varios frentes y que requiere de constantes esfuerzos y tiempo para ser fortalecida (Olaya, 2016). Por ahora la AP debe procurar cumplir con metas de corto plazo que estratégicamente le permita consolidar bases sólidas para la perpetuación de su actividad en el tiempo (Olaya, 2016).

Entretanto, Diego Castillo, encargado de los asuntos económicos y comerciales de la Embajada del Perú en Colombia, considera que los mayores esfuerzos se concentran en el área de los negocios, justamente por el carácter pragmático de la alianza (Castillo, 2016). Los gobiernos involucrados han identificado una alta dependencia de los cuatro países hacia las materias primas, alta presencia de PYMES en las economías nacionales y las altas tasas de informalidad en cada una de ellas (Castillo, 2016). Teniendo en cuenta el alto potencial emprendedor con el que cuenta América Latina, se apostó por la unión de los cuatro mercados en uno más competitivo e integrado que promueva los encadenamientos productivos (Olaya, 2016).

A partir de allí, se pretende fomentar el panorama emprendedor y de innovación de los cuatro Estados para permitir la diversificación del modelo económico actual, que requiere de un aumento de la mano de obra calificada. Todo resulta estar conectado, produciendo una sinergia natural que convierte a la AP en una especie de mega-incubadora de oportunidades para el desarrollo de la región. Una vez fortalecido el ámbito económico y el funcionamiento cooperativo, tendrían mejores herramientas para enfrentar la realidad social de la región. Es en ese momento, cuando el emprendimiento social empezaría a tomar mucha más fuerza (Castillo, 2016).

\section{2. ¿Cómo se aplica lo acordado?: una revisión del proceso de implementación de los mecanismos identificados}

Después de un mapeo del ecosistema de innovación y emprendimiento de la región (AP 2015a), se ha creado una agenda conjunta para incrementar la competitividad y productividad de los cuatro países, con la consolidación de un ecosistema regional de innovación y emprendimiento en la AP (Magendzo y Tefarikis, 
2016). Para ello, el Grupo Técnico de Innovación es el principal encargado de reforzar, mantener y transformar la agenda público-privada en este tema.

Entre los programas que ya están en marcha, hay una red de aceleradoras de negocios y una de inversionistas ángeles de la AP (Magendzo y Tefarikis, 2016): la iniciativa de 'Bien Público Regional', para la articulación conjunta de políticas y programas públicos de expansión de compañías innovadoras (BID, 2016a); el Premio a la Innovación (AP, 2015b); y, la iniciativa de creación de 'Ciudades del conocimiento y valor agregado' (González, 2016).

De manera complementaria, se encuentra en planeación una red de plataformas de crowdfunding, un programa de promoción de la cultura del emprendimiento y la innovación, y el diseño e implementación de instrumentos públicos de internacionalización de las iniciativas emprendedoras e innovadoras (Magendzo y Tefarikis, 2016). Para fomentar tales iniciativas, la AP cuenta con un Fondo de Capital Emprendedor. El BID ha sido uno de sus mayores impulsores, al punto de aprobar, varios millones de dólares en inversiones (BID, 2016b). Además, este será capitalizado con inversiones de los países miembros y aportaciones del sector privado.

El LAB4+ es un espacio de encuentro entre distintos actores, que funciona como laboratorio de ideas relacionadas con el emprendimiento y la innovación; tiene como objetivo impulsarlos "para tener un mayor desarrollo económico, fortaleciendo los lazos entre los cuatro países, así como los encadenamientos productivos y un mejor aprovechamiento de la relación comercial como bloque" (ProColombia, 2014).

Por eso está diseñado para que los gobiernos y el sector privado hagan posible la transferencia de conocimientos y experiencias en estas temáticas (ProColombia, 2014), y generen agendas comunes de investigación que permitan la incubación, desarrollo e internacionalización de emprendimientos y empresas innovadoras de los cuatro países (EFE, 2013). Todo esto, se traduce en esfuerzos por conectar los cuatro ecosistemas de innovación y emprendimiento, y así poder establecer uno que incluya y enfrente los desafíos comunes.

Por su parte, el Premio de Innovación es un mecanismo creado para promover una cultura facilitadora de la innovación entre los países miembros. Una vez institucionalizado, pretende ser un medio para visibilizar las experiencias exitosas. En él participan iniciativas con al menos dos años de impacto en el mercado, que pertenezcan a las categorías de 'Innovación Social', 'TIC', 'Alimentos', 'Educación' y 'Medio Ambiente'. Llama la atención que, aunque 'Innovación Social' premia soluciones novedosas a problemas sociales, la descripción de las otras categorías también incluye cierto grado de impacto social.

Ahora bien, debido a la importancia de la cooperación internacional para la AP, se suscribe el Memorándum de Entendimiento de la Plataforma de Cooperación del Pacífico y el Fondo de Cooperación de la AP. El primero fue firmado en 2011 para impulsar la cooperación entre los cuatro países miembros y con terceros. Entretanto, el segundo facilita, dinamiza y permite la financiación de acciones de cooperación en el marco de la AP.

Finalmente, el Observatorio Regional PYME es un espacio en el que se favorece el acceso a información relevante para emprendedores y PYMES, además de articular las experiencias de cada miembro para el fortalecimiento de sus políticas públicas en estos temas (AP, 2016). Fue propuesta por México a raíz del éxito de su Observatorio Nacional del Emprendedor -ONE- y presentada en la XVI Reunión del Consejo de Ministros en Frutillar en junio del año en curso (AP, 2016).

\section{Conclusiones}

La presente investigación analizó catorce documentos oficiales de la AP, y utilizó estrategias como entrevistas y revisión de diversas fuentes para evidenciar el estado actual de los factores que pueden facilitar la consolidación de procesos de ES, así como sus implicaciones. Todo esto con el objetivo de definir si es posible considerar a la AP como una plataforma para la implementación y promoción del ES en sus países miembros, aun teniendo en cuenta que ambos son expresiones de distintas concepciones de desarrollo.

Para empezar, las marcas de nubes demostraron que los mayores esfuerzos de la AP están dirigidos hacia metas económicas y, se reafirma con el Protocolo Adicional, que justamente se creó para regular dichas dinámicas entre los países miembros. Esto era de esperarse si se tiene en cuenta que la AP se formó a partir de principios neoliberales.

Por su parte, las cuestiones sociales aparecen en repetidas ocasiones, lo que quiere decir que la AP sí presta atención a estos asuntos, en concordancia con la visión de desarrollo que plantea. Sin embargo, al no ser tan recurrentes, significa que ocupan un segundo plano en este marco. De manera complementaria, el análisis de la hoja de codificación, comprueba que la AP puede ser considerada como un elemento activo dentro del regionalismo interdependiente, y de la agenda multidimensional mencionada en el marco teóricoconceptual. Aunque la problemática social sea un factor común, especialmente en los componentes territoriales de las subregiones que conforman la región del pacífico latinoamericano. 
Por otro lado, la codificación y las redes semánticas revelan que los temas de PYMES e innovación ocupan lugares importantes dentro de la AP. Éstos se asocian principalmente con el emprendimiento comercial y su impacto en la dimensión económica del desarrollo. Se observa que la innovación es pieza fundamental en el desarrollo de asuntos económicos, cumpliendo un rol significativo en temas sociales como la educación y las cuestiones medioambientales. Tal interdependencia es precisamente la que caracteriza el proceso de ES.

Teniendo en cuenta que el ES cumple un rol social y otro económico -este último desarrollando un papel bastante similar al de las PYMES-, podría verse beneficiado de las acciones de la AP en dicho campo. Ahora bien, aquí se revela una dificultad, y es que, ninguna de las dos áreas de trabajo son plataformas diseñadas precisamente para el ES, pues se limitan a ser algunas opciones más para encontrar apoyo dentro de la AP.

Al continuar con la sección sobre los mecanismos identificados, se podría afirmar que, hasta el día de hoy, la AP posee o promueve estrategias para la configuración de un ecosistema favorable de ES dentro de sus países miembros. No obstante, la complementariedad e interdependencia resulta ser vital entre sus variables. Por estas razones, lo presentado hasta ese punto no es evidencia suficiente para asegurar que la AP se pueda considerar como plataforma para el ES.

En la investigación se ha insistido en el funcionamiento en red del ecosistema de ES. Para el caso de la AP, todos estos factores son incluidos en los documentos oficiales y se ha podido demostrar que efectivamente se encuentran vinculados entre sí. Adicionalmente, la mayoría de variables del ecosistema se desenvuelven en los tres tipos de áreas de trabajo de la AP, mostrando el carácter multidimensional del ambiente que se está configurando.

Todo se traduce en que, entre compromisos y acciones, la AP brinda apoyo a cada una de las dimensiones y lo hace de manera integrada. Otra observación destacable, es que los mecanismos identificados no fueron creados por la AP en función total del ES; por el contrario, fueron identificados dentro de procesos que abarcan asuntos mucho más amplios, que eventualmente podrían acobijar los procesos de ES.

El último fragmento de este estudio muestra que la AP desde sus inicios ha identificado tanto el potencial emprendedor como el de innovación en América Latina. Así mismo, ha visto la necesidad de apoyarlo en función de la diversificación y fortalecimiento de su modelo de desarrollo. No obstante, también se evidencia que la organización no ha hecho un reconocimiento a la importancia del ES en la región, a pesar de que sus países registran niveles saludables en actividades de este tipo. La tríada "desarrollo/sostenibilidad - capital social y cohesión social" se configuran en variables transversales del ES que debe ser más potencializado en las estrategias de la AP.

Por su parte, las entrevistas y los mecanismos revelan que el desarrollo social se verá impactado de manera positiva, siempre y cuando el sector económico marche como como se ha establecido. Nada particularmente raro, si se recuerda que este es uno de los principios neoliberales sobre los cuales se fundó la AP. Es por esto que no hay un solo por lo que lo documento dedicado exclusivamente al ES.

En resumen, la AP adoptó una agenda pragmática que prioriza las cuestiones económicas. No obstante, ésta no es ajena a las necesidades y problemas que quedan desatendidos bajo dichas dinámicas, por lo que su operación es multidimensional. Esto explica por qué los mecanismos identificados impulsan la productividad y competitividad de las economías involucradas, mientras que incluyen estrategias de doble impacto como el ES y la innovación.

Sin embargo, la AP está lejos de adoptar lógicas concretas del paradigma de desarrollo humano, que permitirían que el ES ocupara una posición más importante dentro del marco de la AP, y así generar mecanismos exclusivos para la instauración de ecosistemas a su favor. No obstante, en la realidad resulta bastante complicado que la AP cambie, especialmente cuando sus miembros creen en los resultados positivos del modelo y son fieles representantes de esos intereses.

De esta manera, el presente estudio logró demostrar que, dentro de las áreas de trabajo y mecanismos diseñados e implementados para abordar dichas temáticas, el ES puede encontrar espacios que facilitan la configuración de un ecosistema que beneficie y soporte su funcionamiento e impactos. Sin embargo, el hecho de que tales instrumentos no estén diseñados específicamente para este tipo de procesos, hace que su impulso por parte de la AP resulte insuficiente y, en ciertos casos, pueda representar un obstáculo más que los emprendedores sociales deben sortear dentro de los países miembros.

\section{Referencias bibliográficas}

Agencia Española de Cooperación Internacional para el Desarrollo (2015) La integración regional en América Latina. Madrid: Cooperación Española.

Alianza del Pacífico (2012a) Abecé Alianza del Pacífico. Obtenido de Alianza del Pacífico: https://alianzapacifico.net/que-es-la-alianza/.

Alianza del Pacífico (2012b) Temas de Trabajo. Obtenido de Alianza del Pacífico: https://alianzapacifico.net/temas-detrabajo/. 
Alianza del Pacífico (2012c) ¿Qué es el Observatorio Regional PYME Alianza del Pacífico? Obtenido de Observatorio Regional PYME: pymes/index.php/component/content/article?id=68.

Alianza del Pacífico (2015a) XXII Ronda de Reuniones de los Grupos Técnicos de la Alianza del Pacífico. Obtenido de Alianza del Pacífico: https://alianzapacifico.net/xxii-ronda-de-reuniones-de-los-grupos-tecnicos-de-la-alianza-delpacifico/.

Alianza del Pacifico (2015b) La AP fortalece sus ecosistemas de innovación. Obtenido de Alianza del Pacífico: https://alianzapacifico.net/la-alianza-del-pacifico-fortalece-sus-ecosistemas-de-innovacion/.

Alianza del Pacífico (2016) XVI Reunión del Consejo de Ministros en Frutillar. Obtenido de Alianza del Pacífico: https://alianzapacifico.net/xvi-reunion-del-consejo-deministros-se-reune-en-frutillar/.

Arksey, H., \& Knight, P. (1999) Interviewing for social scientists - an introductory resource with examples. Londres: SAGE Publications.

Avogadro, E. (2016) Is Latin America ready for a creative economy? Obtenido de World Economic Forum: https://www.weforum.org/agenda/2016/06/is-latin-america-ready-for-a-creative-economy.

BID (2016a) BPR apoyará ecosistema de innovación de la Alianza del Pacífico. Obtenido de Competitividad, Tecnología e Innovación: http://www.iadb.org/es/temas/competitividad-tecnologia-e-innovacion/bpr-apoyaraecosistema-de-innovacion-de-la-alianza-del-pacifico, 19226.html.

BID (2016b) FOMIN invierte en nuevo fondo de capital para empresas innovadoras en la Alianza del Pacífico. Obtenido de Comunicados de Prensa: http://www.iadb.org/es/noticias/comunicados-de-prensa/2016-08-11/fomininvierte-en-fondo-para-empresas-de-la-ap,11535.html.

Betancourt, R., y Prieto, G. (2014) Entre la soberanía, el liberalismo y la innovación - un marco conceptual para el análisis de la Alianza del Pacífico. En E. Pastrana Buelvas, \& H. Gehring, Alianza del Pacífico: mitos y realidades, pp. 75-113. Bogotá: Universidad Santiago de Cali.

Bosma, N., Schøtt, T., Terjesen, S., \& Kew, P. (2015) Global Entrepreneurship Monitor Special Topic Report - Social Entrepreneurship. Londres: Global Entrepreneurship Research Association, London Business School.

Briceño-Ruiz, J. (2014) Del regionalismo abierto al regionalismo poshegemónico en América Latina. En W. SotoAcosta, Política internacional e integración regional comparada en América Latina, pp. 23-34. San José: Universidad Nacional de Costa Rica/FLACSO.

Briceño-Ruiz, J., \& Morales, I. (2017) Open regionalism and post-hegemonic regionalism in the Americas contextualizing the debate. En J. Briceño-Ruiz, \& I. Morales, Post-hegemonic regionalism in the Americas, pp. 128. Nueva York: Routledge.

Buckland, H., \& Murillo, D. (2014) La innovación social en América Latina - marco conceptual y agentes. Washington, D.C.: Instituto de Innovación Social de ESADE y Fondo Multilateral de Inversiones.

Cancino, A., \& Albornoz, C. (2007) La integración regional como instrumento de desarrollo para América Latina. Colombia Internacional, $\mathrm{N}^{\circ} 66$, pp. 120-146.

Cassar, G. (2015) How can Latin America achieve long-term inclusive growth? Obtenido de World Economic Forum: https://www.weforum.org/agenda/2015/05/how-can-latin-america-achieve-long-term-inclusive-growth.

Castillo, D. (2016) Comunicación Personal. (A. Figueroa, Entrevistador), 4 de Noviembre de 2016.

Chen, J., Saarenketo, S., \& Puumalainen, K. (2016) Internationalization and value orientation of entrepreneurial ventures - a Latin American perspective. Journal of International Entrepreneurship, № 14, Vol. 1, pp. 32-51.

Dees, G. (2011) El significado del "emprendimiento social". Revista Española del Tercer Sector, No 17, pp. 111-124.

Echebarría, L. y Estevadeordal, A. (2014) Alianza del Pacífico - un nuevo motor de integración regional. En A. Foxley, \& P. Meller, Alianza del Pacífico - en el proceso de integración latinoamericana, pp. 27-42. Santiago de Chile: Cieplan.

EFE (2013) El I Foro de Innovación y Emprendimiento de la Alianza del Pacífico comienza el miércoles. Obtenido de EFE: Emprende: http://www.efeemprende.com/noticia/el-i-foro-de-innovacion-y-emprendimiento-de-la-alianza-delpacifico-comienza-el-miercoles/.

Farrell, M., Hettne, B., \& Van Langenhove, L. (2005) Global politics of regionalism - theory and practice. Londres: Pluto Press.

Fernández de Soto, G. (2015) La Alianza del Pacífico - un ejemplo para la integración regional. Actualidad Jurídica Uría Menéndez, № 39, pp. 7-18.

Flemes, D. y Radseck, M. (2012) Gobernanza multinivel de seguridad en América del Sur. Papel Político, No 17, Vol. 1, pp. 203-238.

Forero, M. (2010) Orígenes, problemas y paradojas de la integración latinoamericana. Revista Análisis Internacional, $\mathrm{N}^{\circ} 2$, pp. 155-182.

Foxley, A. y Meller, P. (2014) Alianza del Pacífico - en el proceso de integración latinoamericana. Santiago de Chile: Cieplan.

Gamboa, F. y Fernández, M. (2013) Tratado de Derecho Internacional Público y Derecho de Integración. Santiago de Chile: Thomson Reuters.

González, L. (2016) Alianza del Pacífico trabaja en innovación. Obtenido de El Economista: http://eleconomista.com.mx/industrias/2016/03/15/alianza-pacifico-trabaja-innovacion.

Guzmán, A. y Trujillo, M. (2008) Emprendimiento social - revisión de literatura. Estudios Gerenciales, № 24 , Vol. 109 , pp. 105-126. 
Hernández, R., Fernández, C. y Baptista, P. (2014) Metodología de la investigación. Ciudad de México: Interamericana Editores.

Hopenhayn, M (2007) Cohesión social - una perspectiva en proceso de elaboración. En: A. Sojo, \& A. Uthoff, Cohesión social en América Latina y El Caribe - una revisión perentoria de algunas dimensiones, pp. 35-47. Nueva York, Naciones Unidas, CEPAL.

Jordana, J. (2000) Instituciones y capital social. ¿Qué explica qué? Revista Española de Ciencia Política, № 1, Vol. 2, pp. 187-210.

Lejarriaga Pérez de las Vacas, G. y Bel Durán, P. (2018) Emprendimiento, emprendimiento social, emprendimiento en economía social y emprendimiento a través de organizaciones de participación. Presentado en las $\mathrm{V}$ Jornadas de investigación y docencia en materia de empresas de participación. Emprendimiento social y nuevas formas de hacer economía: su relación con las organizaciones de participación y la economía social celebradas el 16 de febrero de 2018, en Madrid.

López, A. (2016) América Latina le apuesta al emprendimiento social. Obtenido de TecReview: http://tecreview.itesm.mx/america-latina-le-apuesta-al-emprendimiento-social/.

Luna, J. (2012) Influencia del capital humano para la competitividad de las PYMES en el sector manufacturero de Celaya, Guanajuato. Celaya: Universidad de Celaya.

Magendzo, A. y Tefarikis, E. (2016) El virus que crece en la Alianza del Pacífico. Obtenido de Puntos Sobre la i - un blog del BID para conversar sobre innovación en América Latina y el Caribe: http://blogs.iadb.org/puntossobrelai/2016/07/15/el-virus-que-crece-en-la-alianza-del-pacifico/.

Machinea, J.L. (2007) El financiamiento solidario de la protección social. Condición indispensable para un pacto de cohesión social en América Latina. En: A. Sojo, \& A. Uthoff, Cohesión social en América Latina y El Caribe - una revisión perentoria de algunas dimensiones, pp. 23-34. Nueva York, Naciones Unidas, CEPAL.

Martín López, S. (2018) ¿Cómo se concreta “la creación de valor social sostenible” en las iniciativas de emprendimiento social? Ponencia presentada en el XII Congreso Internacional de Rulescoop. Brest, 22 al 24 de Mayo de 2018.

Moreno, C. (2016) Comunicación Personal. (A. Figueroa, Entrevistador), 28 de Octubre de 2016.Max-Neef, M. (1986). Desarrollo a esacala humana - una opción para el futuro. Santiago de Chile: CEPAUR.

Nye, J. (1968) International regionalism - readings. Boston: Little, Brown and Company.

Olaya, C. (2016) Comunicación Personal. (A. Figueroa, Entrevistador), 01 de noviembre de 2016.

Pastrana, E., Betancourt, R. y Simmonds, O. (2014) Regionalización, regionalidad y gobernanza multinivel en Suramérica. En: E. Pastrana, \& H. Gehring. Suramérica en el escenario global - gobernanza multinivel y birregionalismo, pp. 85-123. Bogotá, D.C.: Fundación Konrad Adenauer Stiftung.

Pastrana, E. (2015) La Alianza del Pacífico - de cara a los proyectos regionales y las transformaciones globales. Bogotá: Fundación Konrad Adenauer.

Payne, A. (2004) The new regional politics of development. Nueva York: Palgrave.

Petersmann, E.-U. (2017) Globalization and multi-level governance. En T. Cottier, \& K. Nadakavukaren Schefer, Elgar Encyclopedia of International Economic Law, pp. 39-40. Cheltenham: Edward Elgar.

ProColombia (2014) Asociación, la clave del emprendimiento e innovación - Lab4 de Alianza del Pacífico. Obtenido de Noticias de Procolombia: http://www.procolombia.co/noticias/asociacion-la-clave-del-emprendimiento-einnovacion-lab4-de-alianza-del-pacifico.

Putnam, R. (1993) Making democracy wor - civic traditions in modern Italy. Princeton: Princeton University Press.

Reza Noruzi, M. (2010) An exploration of social entrepreneurship in the entrepreneurship era. Asian Social Science, ${ }^{\circ}$ 6, Vol. 6, pp. 3-10.

Rosales, O. (2012) El vínculo económico y comercial de América Latina con China después del discurso de Wen Jiabao. En F. Reyes Matta, O. Rosales, \& J. Valenzuela, China-América Latina: ¿Cómo ir más allá del 2020?, pp. 111-140. Santiago de Chile: Centro de Estudios Latinoamericanos sobre China, Universidad Andrés Bello.

Sánchez Espada, J., Martín López, S., Bel Durán, P. y Lejarriaga Pérez de las Vacas, G. (2018) Educación y formación en emprendimiento social - características y creación de valor social sostenible en proyectos de emprendimiento social. REVESCO. Revista de Estudios Cooperativos, Tercer Cuatrimestre, $\mathrm{N}^{\mathrm{o}} 129$, pp. 16-38. DOI: 10.5209/REVE.62492.

Santos, F. (2009) A positive theory of social entrepreneurship. Fontainebleau: INSEAD.

Stephan, U., Uhlaner, L., \& Stride, C. (2015) Institutions and social entrepreneurship - the role of institutional voids, institutional support, and institutional configurations. Journal of International Business Studies, No 46, Vol. 3, pp. 308-331.

Thoene, U., \& Gaitán-Guerrero, L.A. (2019) Competition law and policy in the regional context - European Union experiences for the Pacific Alliance. En P. Sauvé, R. Polanco Lazo, \& J.M. Álvarez Zárate, The Pacific Alliance in a world of preferential trade agreements, pp. 215-233. Basilea: Springer.

Thoene, U., Cuestas-Zamora, E.J., y Londoño-Lázaro, M.C. (2017) La Alianza del Pacífico y el regionalismo latinoamericano - en búsqueda de una revitalización autonomista de la integración. Análisis Político, № 30, Vol. 89, pp. 91-110.

Thoene, U., y Turriago-Hoyos, A. (2017) Financial inclusion in Colombia - a scoping literature review. Intangible Capital, $\mathrm{N}^{\mathrm{o}}$ 13, Vol. 3, pp. 582-614. 
Tolama, J. (2016) Chile, Colombia, Perú y México, ¿Qué hacen por los emprendedores? Obtenido de Expansión en alianza con CNN: http://expansion.mx/emprendedores/2016/10/07/chile-colombia-peru-y-mexico-que-hacen-porlos-emprendedores.

U1 Haq, M. (1995) Reflections on human development. Oxford: OUP.

Ul Haq, M. (2018a) El paradigma del desarrollo humano. Alianza Latinoamericana de estudios críticos para el desarrollo. En http://estudioscriticosdesarrollo.com/desarrollohumano/MulHaqParadigmaDesarrolloHumano.pdf.

Ul Haq, M. (2018b) Economic Growth with social justice. Oxford: OUP.

Vernis, A. y Navarro, C. (2011) El concepto de ecosistemas para el emprendimiento social. Revista Española del Tercer Sector, No 17 , pp. 67-86.

Vieira, E. (2007) La formación de espacios regionales en la integración de América Latina. Bogotá: Pontificia Universidad Javeriana.

Vivares, E. y Calderón, M. (2014) Desarrollo, poder y regionalismo sudamericano. Obtenido de FLACSO: http://www.planificacion.gob.ec/wp-content/uploads/downloads/2014/08/Desarrollo-poder-y-regionalismoSudamericano-Vivares-Calderon.pdf.

Zuñiga, F. (2016) ¿Cuál es el panorama del emprendimiento social en América Latina? Obtenido de América Economía: http://mba.americaeconomia.com/articulos/entrevistas/cual-es-el-panorama-del-emprendimiento-socialen-america-latina. 